Classification

Physics Abstracts

$07.80-81.00$

\title{
Some TEM investigations on composites, alloys-superalloys and superconductive ceramics
}

\author{
Jean Galy, Jean Sevely, Daniel Caillard and Christian Roucau \\ Centre d'Elaboration de Matériaux et d'Etudes Structurales, Laboratoire d'Optique Electronique, \\ CEMES-LOE/CNRS, BP 4347, 31055 Toulouse Cedex, France
}

(Received October 01, 1990; accepted January 25, 1991)

\begin{abstract}
Résumé. - Composites • L'étude des polytypes SiC par HREM, les analyses chimiques par EELS et les structures fines par EXELFS ont été réalisées sur des composites SiC-SiC. Alliages-Superalliages - Des expériences de déformation in situ dans un microscope électronique à transmission sur $\mathrm{Be}$, $\mathrm{Ni}_{3} \mathrm{Al}$ et des superalliages sont rapportées. Céramiques supraconductrices $\bullet$ Le microscope à haute tension ( $3 \mathrm{MeV}$ ), équipé d'une microchambre, a permis l'observation directe de la transformation quadratique $\leftrightarrow$ orthorhombique des phases supraconductrices $(\mathrm{Ln}) \mathrm{Ba}_{2} \mathrm{Cu}_{3} \mathrm{O}_{6+x}(0 \leq x \leq 1)(\mathrm{Ln}$ "cocktail" de terres rares $\mathrm{Y}, \mathrm{Nd}, \mathrm{Sm}, \mathrm{Eu}, \mathrm{Yb})$. L'influence de la substitution de terres rares au calcium dans les phases $\mathrm{Bi}-\mathrm{Sr}-\mathrm{Ca}-\mathrm{Cu}-\mathrm{O}$, qui affecte $T_{\mathfrak{c}}$, a eté suivie au niveau structural par des investigations combinées aux rayons $\mathrm{X}$ et en microscopie électronique.
\end{abstract}

\begin{abstract}
Composites - HREM study, chemical analysis by EELS and fine structure by EXELFS have been realized on $\mathrm{SiC}$ polytypes existing in SiC-SiC composites. Alloys-Superalloys • TEM in situ straining experiments performed on $\mathrm{Be}, \mathrm{Ni}_{3} \mathrm{Al}$ and superalloys are reported. Superconductive ceramics $\bullet$ HVEM ( $3 \mathrm{MeV}$ ) equipped with a gas microchamber has allowed the direct observation of the tetragonal $\leftrightarrow$ orthorhombic transition of the superconductive phases $(\mathrm{Ln}) \mathrm{Ba}_{2} \mathrm{Cu}_{3} \mathrm{O}_{6+x}(0 \leq x \leq$ 1) (Ln "cocktail" of rare earth $\mathrm{Y}, \mathrm{Nd}, \mathrm{Sm}, \mathrm{Eu}, \mathrm{Yb}$ ). Influence of the rare earth substitution for $\mathrm{Ca}$ in $\mathrm{Bi}-\mathrm{Sr}-\mathrm{Ca}-\mathrm{Cu}-\mathrm{O}$, which affects $T_{\mathfrak{c}}$, is also reported.
\end{abstract}

\section{Introduction.}

The main scientific lines of the new CNRS laboratory, the Centre d'Elaboration de Matériaux et d'Etudes Structurales built on the basis of the Laboratoire d'Optique Electronique, are devoted to the structure and microstructure of materials with a view to deepening our understanting of the relation between their chemical and physical properties and the atomic architecture.

We choose to develop here three applications of TEM techniques:

- a joint investigation by TEM and EELS techniques of composites with ceramic matrix;

- the study of alloys-superalloys by in situ TEM observations;

- the study of superconductive $\mathrm{YBaCuO}$ and $\mathrm{BiSr}(\mathrm{CaLn}) \mathrm{CuO}$ families by TEM and HVTEM (3 MV microscope, gas microchamber). 


\section{Composites.}

In recent years there has been considerable interest in the study of silicon carbide which appears as an important element in the development of ceramic composite materials.

Siiicon carbide exists as a number of different structural types commonly called polytypes. These polytypes correspond to variations of a one-dimensional stacking sequence. The existence of the so-called "one dimensionally disordered" structure in SiC is associated with a particular distribution of these polytypes as well as planar defects generic to these structures. The various degrees of randomness in the assemblage of these structures reflect the thermal and mechanical history of the material. There is thus a high interest in the determination of these arrangements at an atomic level.

It has been shown $[1,2]$ that high resolution electron microscopy (HREM) combined with electron diffraction provides excellent means for the characterization of polytypes and enables the recognition and detailed analysis of defects in these structures: dislocations, stacking faults, antiphase and domain boundaries, etc. Due to the high scattering power of electrons, the data can be obtained on very small quantities of materials (lower than $0.1 \mu \mathrm{m}$ scale). The coupling of these techniques to the microanalysis facilities associated with electron energy loss spectroscopy (EELS) enables an accurate determination of the local chemical composition of the material. A further and specific development of this technique allows the measurement of the local atomic order around each selected atomic species from their extended energy-loss fine structure (EXELFS) [3]. In this way the addition of these techniques may contribute to a larger understanding of the relationship between structure and mechanical properties of these materials. Using HREM and EELS, we have performed such a study in the case of various silicon carbide specimens having different history.

2.1 SiliCON CARBIDE POLYTYPE STRUCTURE. - The fundamental unit in all SiC polytypes is the coordination tetrahedron in which a silicon atom is surrounded by four carbon atoms (or similarly a carbon atom is surrounded by four silicon atoms), figure 1 . These tetrahedra are stacked in positions analogous to those occupied by spheres in close-packed structure and linked through their apices to form a tetrahedral covalently bonded, polar structure. Two stacking sequences are found in silicon carbide: a simple cubic $(\mathrm{ABC})$ structure, due to a translation only of adjacent layers and both translation and rotation, yielding $\mathrm{AC} \mathrm{AC} \mathrm{sequences} \mathrm{corresponding} \mathrm{to} \mathrm{an} \mathrm{hexag-}$ onal stacking. (According to Ramsdell [4] the notation $n \mathrm{C}, n \mathrm{H}, n \mathrm{R}$ may be used to describe all polytypes. The number $n$ denotes the number of layers in one unit cell while the letter denotes the Bravais-lattice type (cubic, hexagonal, rhombohedral)).

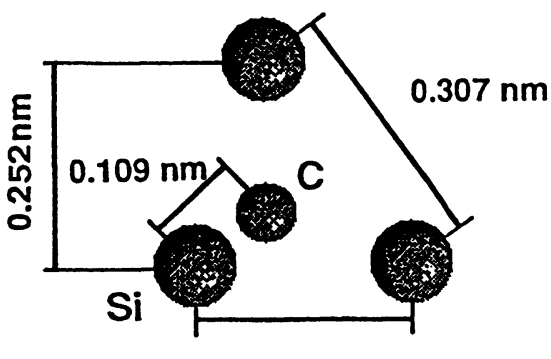

$0.266 \mathrm{~nm}$

Fig. 1. - Projection of the SiC coordination tetrahedron in the $<1120>$ direction (hexagonal stacking) or $<110>$ direction (cubic). 
2.2 SILICON CARBIDE H.R. IMAGING - Every long period polytype of SiC has a structure which is simply some periodic stacking of subunits cells.

When silicon carbide is observed, in order to reveal the stacking sequence, the structure has to be viewed along a direction perpendicular to the $c$-axis and in order to obtain subunit cell information the crystal should be orientated preferentially along $<11 \overline{2} 0>$ direction for the hexagonal stacking or $<110>$ for the cubic polytype $[1,2,5]$.

For the polytype characterization the multiple beam imaging mode and high magnification $(>400000 \mathrm{X})$ are required. The general procedure for the image interpretation is the correlation of the calculated image with the experimental one in a given projection direction.

The image calculations, required to gain confidence in high resolution image interpretation, implies [6]:

1) to treat the information in terms of multiple beam dynamical diffraction theory,

2) to take account of lens aberration effect on the information transfer in the microscope.

These calculations are made by using the MAC TEMPAS program deduced from the SHRLI program developped by O'Keefe and Buseck [7]. By superimposing the projection of the structure calculated by this way with the image, it is possible to correlate the position of the atomic rows with the contrast distribution in the image. Figure 2 shows an example of this correlation in the case of a $6 \mathrm{H}$ silicon carbide structure, observed at $200 \mathrm{kV}$ with a thickness $t=20 \mathrm{~nm}$ for a defocus condition of the objective lens $\Delta z=-89 \mathrm{~nm}$.

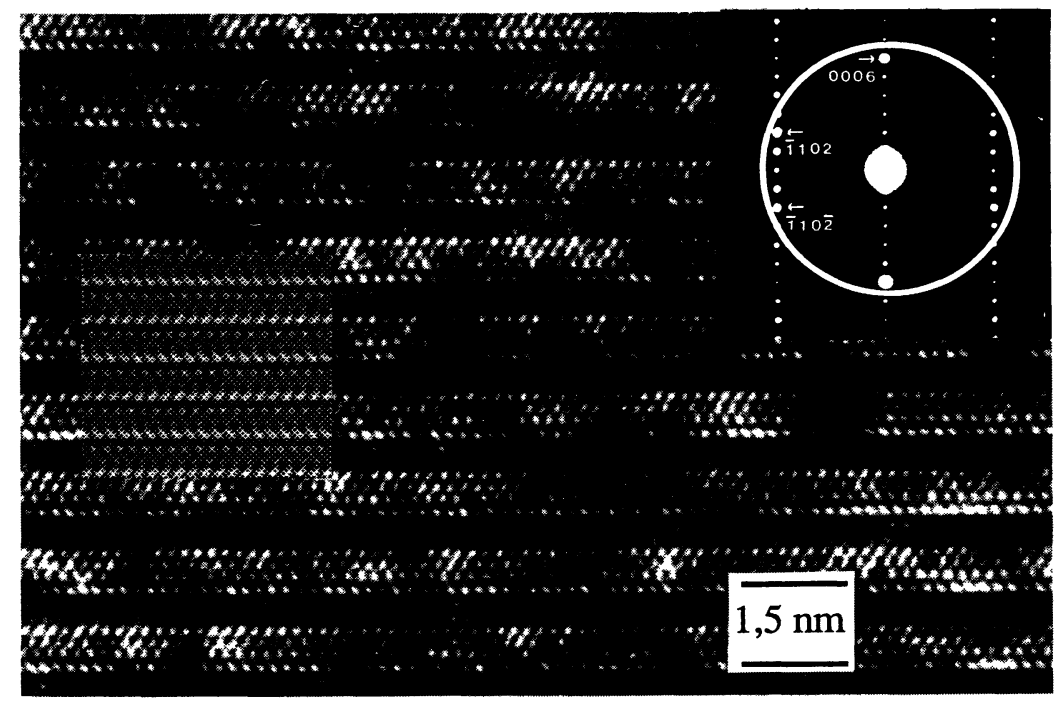

Fig. 2. - Experimental and simulated (superimposed) lattice images of $6 \mathrm{H} \mathrm{SiC}$ single crystal at $200 \mathrm{kV}$ and associated diffraction pattern.

This image clearly shows the $1.5 \mathrm{~nm}(6 \times 0.252 \mathrm{~nm})$ periodic distribution of the intensity in the image. This distribution is characteristic of the unit-cell spacing fringes of the $6 \mathrm{H}$ silicon carbide structure. In the same way the calculated and experimental images show the zig-zag stackings, $3-3$, of the planes $(\overline{1102})$ and $(\overline{1} 10 \overline{2})$ which are characteristic of the $6 \mathrm{H}$ polytype.

In the case of more disordered material, as the one observed in composite materials, the same technique is applicable to the study of polytype distribution and transformation mechanisms. This 
has been made for SiC polytype prepared by chemical vapor infiltration [5]. These examples show that HREM imaging coupled with electron diffraction and image simulation provides us with excellent means for the characterization of polytypes, particularly information on stacking sequences of atomic planes in the material.

Generally, the manufacture processings of the material introduce some impurities in the material. The addition of electron energy loss spectroscopy allows us to characterize artefacts in the material by the detection of chemical elements and the determination of the short range order around the silicon or carbon atomic sites.

\subsection{ELECTRON ENERGY LOSS SPECTROSCOPY (EELS).}

\subsubsection{Chemical characterization and quantitative analysis.}

2.3.1.1 Principle. - Thin parts of the SiC sample (thickness $t$ ranging from 10 to $100 \mathrm{~nm}$ ), suitable for transmission electron microscopy, were observed at $120 \mathrm{keV}$ with a Philips EM400T electron microscope, equipped with a VG (ELS 80) electron energy loss spectrometer. For the electron energy loss studies the spectra were associated to a volume of the sample, which is defined by the probe size of the electron beam on the sample $15 \mathrm{~nm}$ in diameter and the thickness $t$ of the specimen at this point. The energy loss spectra bring information on electron-electron inelastic interactions in the sample. This information associated with the inner shell electron excitation by the incident electrons is considered for the elemental analysis of the material.

2.3.1.2 Quantitative analysis method. - The silicon concentration relative to carbon, was determined by the classical quantitative analysis technique of the EEL spectra, as proposed by Egerton or Isaacson for instance $[9,15]$. The technique has been established through the assumption that for low and medium atomic number elements, the concentration $N^{\mathrm{A}}$ of an element A can be calculated through the use of the formula:

$$
N^{\mathrm{A}}=\frac{1}{\sigma_{k}^{\mathrm{A}}(\alpha, \Delta)} \cdot \frac{I_{k}^{\mathrm{A}}(\alpha, \Delta)}{I_{1}(\alpha, \Delta)}
$$

Where $I_{k}^{\mathrm{A}}(\alpha, \Delta)$ and $I_{1}(\alpha, \Delta)$ are integrated intensities within the energy loss spectrum (Fig. 3) under the profile associated to the $k$-shell excitation and the low energy loss distribution, respectively; $\sigma_{k}^{\mathrm{A}}(\alpha, \Delta)$ is the partial cross section for the $k(k=\mathrm{K}$ or $\mathrm{L})$ inner shell excitation of the element $\mathrm{A}, \alpha$ is the collection semi-angle in the microscope, while $\Delta$ is the energy loss window on which the intensity is measured in the spectra.

The ratio $N^{\mathrm{A}} / N^{\mathrm{B}}$ of the concentration of two elements $\mathrm{A}$ and $\mathrm{B}$, characterized by their inner shell excitation intensities $I_{k}^{\mathrm{A}}$ and $I_{k}^{\mathrm{B}}$ respectively, when they are measured on the same spectrum, is given by rewriting this equation under the form:

$$
\frac{N^{\mathrm{A}}}{N^{\mathrm{B}}}=\frac{\sigma_{k}^{\mathrm{B}}(\alpha, \Delta) I_{k}^{\mathrm{A}}(\alpha, \Delta)}{\sigma_{k}^{\mathrm{A}}(\alpha, \Delta) I_{k}^{\mathrm{B}}(\alpha, \Delta)}
$$

The same integration range $\Delta$ has to be used for the measurement of the intensity, so that the determination of $I_{k}(\alpha, \Delta)$ and the calculation of the partial cross-section $\sigma_{k}(\alpha, \Delta)$ for the two elements may led to a measure of their relative concentration value in a chosen microvolume of the sample.

These expressions have a limited range of validity. Due to multiple scattering effects of the electrons in the sample, the shape of the characteristic excitation profiles changes when the thickness $t$ increases. It has been shown that these equations are accurate only up to the thickness $t / \lambda<1$ in the case of boron nitride $[9,10]$ for instance ( $\lambda$ being the mean free path for inelastic scattering of the incident electron in the sample). It can be considered that this approximation is valid to within $10 \%$ [11]. 


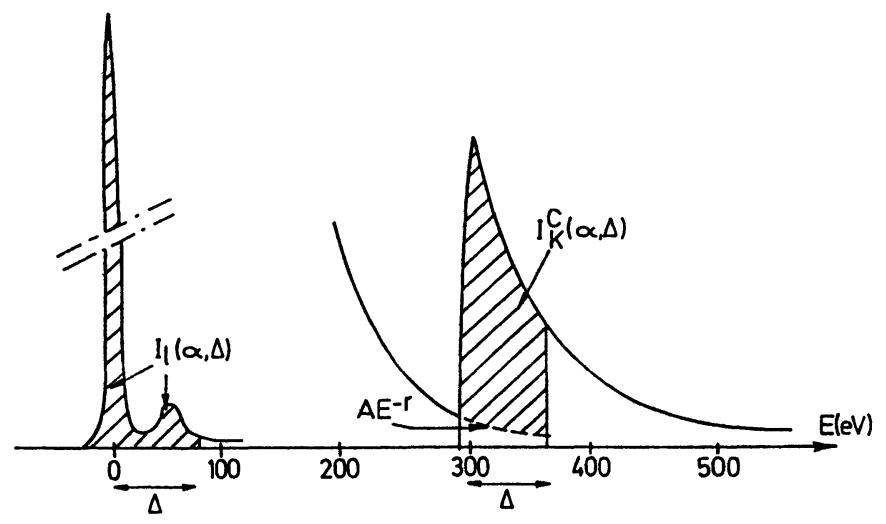

Fig. 3. - Example of an electron energy loss spectrum with the definition of the main analysis parameters for the elemental quantitative analysis.

2.3.1.3 Measurement. - Generally we have chosen the thickness of the section in such a way that $t / \lambda=0.5$. That means $t=500-700 \AA$ thick sections of the specimen. For the characterization of carbon and silicon the spectra were recorded in the energy loss range $200-2,000 \mathrm{eV}$. This range contains the carbon and silicon $\mathrm{K}$ shell excitation edges at 285 and $1840 \mathrm{eV}$, respectively (Fig. 4).

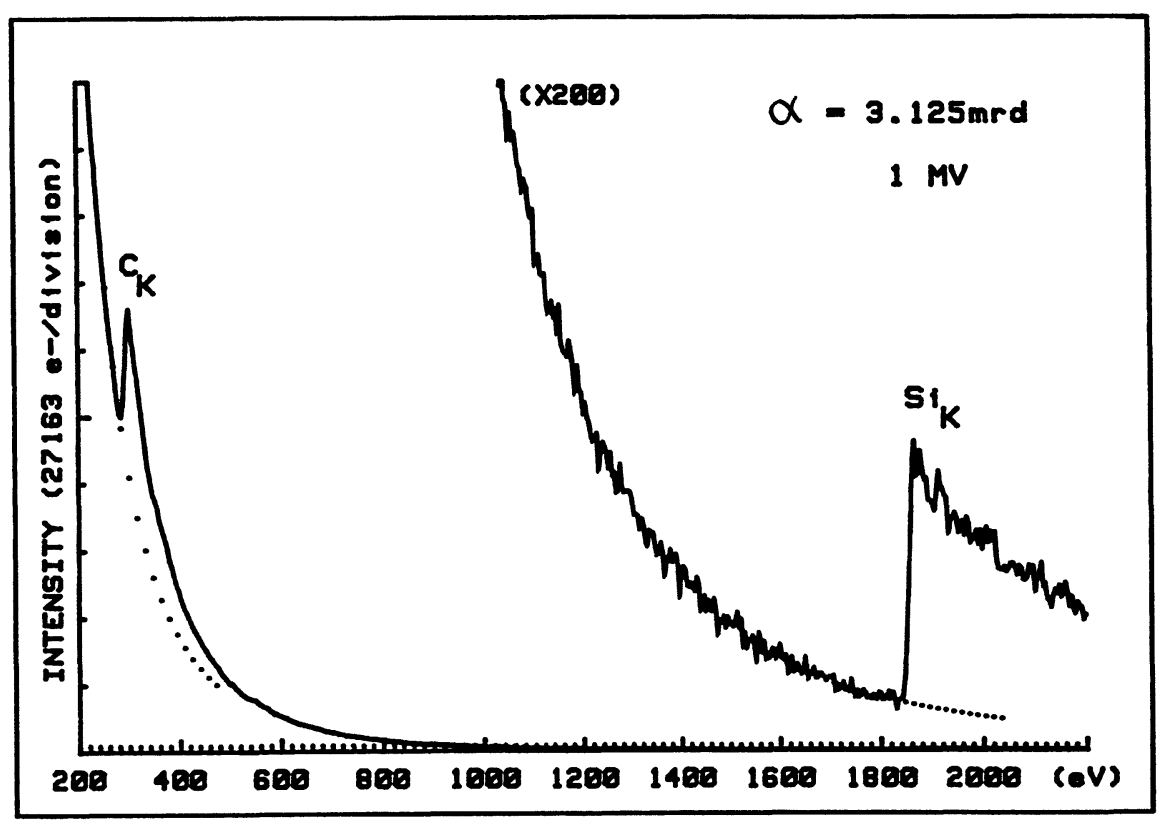

Fig. 4. - EEL spectrum showing the K excitation edges of the carbon and the silicon with the background fitting in order to determine the core-loss integrals $I_{k}^{\mathrm{C}}(\alpha, \Delta)$ and $I_{k}^{\mathrm{Si}}(\alpha, \Delta)$. 
As an example of this measurement for the spectrum of figure 4 obtained with $1 \mathrm{MV}$ electrons when $\Delta$ is $150 \mathrm{eV}$ and the collection angle $\alpha=3.13 \mathrm{mrad}$. The following values are:

for carbon: $I_{k}^{\mathrm{C}}(3.13 \mathrm{mrad}, 150 \mathrm{eV})=1,229,232 \mathrm{cts}, \sigma_{k}^{\mathrm{C}}(3.13 \mathrm{mrad}, 150 \mathrm{eV})=$ $3.653 \times 10^{-21} \mathrm{~cm}^{2}$ and for silicon: $I_{k}^{\mathrm{Si}}(3.13 \mathrm{mrad}, 150 \mathrm{eV})=14,371 \mathrm{cts}, \sigma_{k}^{\mathrm{Si}}(3.13 \mathrm{mrad}, 150 \mathrm{eV})=$ $4.239 \times 10^{-23} \mathrm{~cm}^{2}$. With these values the ratio $N^{\mathrm{Si}} / N^{\mathrm{C}}=1.007$ which confirms that the stoichiometry of $\mathrm{SiC}$ is preserved in this part of the material.

\subsection{EXELFS ANALYSES OF SiC.}

Principle. - Extended electron energy loss fine structure (EXELFS) is a technique which is sensitive to the local environment surrounding low $Z$ element atoms, including Si and $\mathrm{C}$. Analyses of EXELFS modulations present on core loss edges have been performed with the use of the Philips EM 400 microscope and with a High Voltage (1 MV) Electron Microscope (HVEM). Some of the advantages for using higher accelerating voltages in Electron Energy Loss Spectroscopy (EELS) are the greater specimen penetration and improved signal to noise ratio which can lead to improved analyses of both low or high $Z$ containing materials [12]. The modulations observed on the core edges of EELS spectra are related to the same type of interference also found on X-ray absorption edges known as EXAFS (Extended X-ray Absorption Fine Structure). They result from interaction of ejected core electrons with neighboring atoms and they can be processed in the same way [13].

The materials used for this study included single crystal SiC and some part of single crystal $\mathrm{SiC}$ made amorphous by ion implantation. The EXELFS results, are based upon the carbon and silicon K edges observed in the spectra [14].

High quality spectra from typically $100 \mathrm{~nm}$ regions where recorded in the image mode (diffraction coupled) with a collection angle ranging from 3 to $10 \mathrm{mrad}$ and $t / \lambda$ values between 0.2 and 0.4 .

A typical EXELFS analysis requires various operations on the data, including: (1) background subtraction, (2) determination of the EXELFS oscillation function, $\chi$, by fitting a fourth or fifth degree polynomial, (3) conversion of the energy scale to the momentum $(k)$ space, (4) use of a cubic spline interpolation routine to provide $2^{n}$ evenly spaced points, (5) application of a window function (consisting of a constant value centered in the $k$ range and decreasing by a cosine function to zero on the ends) to the $k_{\chi}(k)$ data, (6) extension of the effective $k$ range by a factor of eight by adding zeros to the data set and (7) determination of the modulus (power spectrum) of the fast fourier transform. The power spectrum is related to the radial distribution function, RDF, and plotted versus the real space distance.

2.5 RESULTS [14]. - An EELS spectrum of the Si K absorption edge for crystalline SiC was measured and the resulting EXELFS oscillation function, $\chi$, was weighted by the momentum transfer variable, $k$. The Fourier transform was taken and the resulting radial distribution function is shown in figure 5, where the results are compared for crystalline and amorphous materials deduced from the silicon K edge. The first peak in the RDF corresponds to the Si-C interatomic distance. The measured value is $1.37 \AA$ compared to the known value of $1.88 \AA$. This implies that a correction factor of $0.51 \AA$ is needed, whereas the calculated phase shift based on $3<k<8 \AA^{-1}$ is $0.50 \AA$. The second peak, at $2.63 \AA$, corresponds to the Si-Si distance. The known distance is $3.08 \AA$ A phase shift correction of $0.45 \AA$ is thus required in good agreement with a calculated value of $0.45 \AA[3]$.

Transmission electron microscopy (TEM) analyses of cross-sectional, ion implanted SiC specimens revealed an amorphous surface layer of $\approx 1700 \AA$ thickness. Various EELS spectra were taken of the Si K edge from SiC. amorphized by ion implantation. The resulting EXELFS oscilla- 
tion function, $\chi$, was again weighted by $k$ and the Fourier transform obtained. The resulting RDF is shown in figure $6 \mathrm{~b}$. The first peak in the RDF occurs at $1.54 \AA$. Applying the phase shift correction factor determined from the crystalline $\mathrm{SiC}$ of $0.51 \AA$ results in a corrected Si-C interatomic distance of $2.04 \AA$. The second peak in the RDF occurs at $2.93 \AA$ and should correspond to the $\mathrm{Si}-\mathrm{Si}$ interatomic distance. Applying the measured phase shift correction factor determined from the crystalline $\mathrm{SiC}$ results in a value of $3.33 \AA$.

The same treatment is applied on the carbon Kedge in the two types of materials, figure 6 . The first peak positions in the RDF, corresponding to the first C-Si distance, is measured at $1.49 \AA$ in the crystalline material and $1.66 \AA$ for the amorphous one. Applying the phase shift correction factor: $0.38 \AA$, deduced from Teo and Lee tables [16] they correspond to 1.87 and $2.04 \AA$ respectively. A weak second peak in the RDF can be attributed to a C-C distance, taking account of a calculated phase shift correction $0.4 \AA$ they give a C-C distance of $3.03 \AA$ in the crystalline material and $3.34 \AA$ in the material made amorphous by ion implantation.
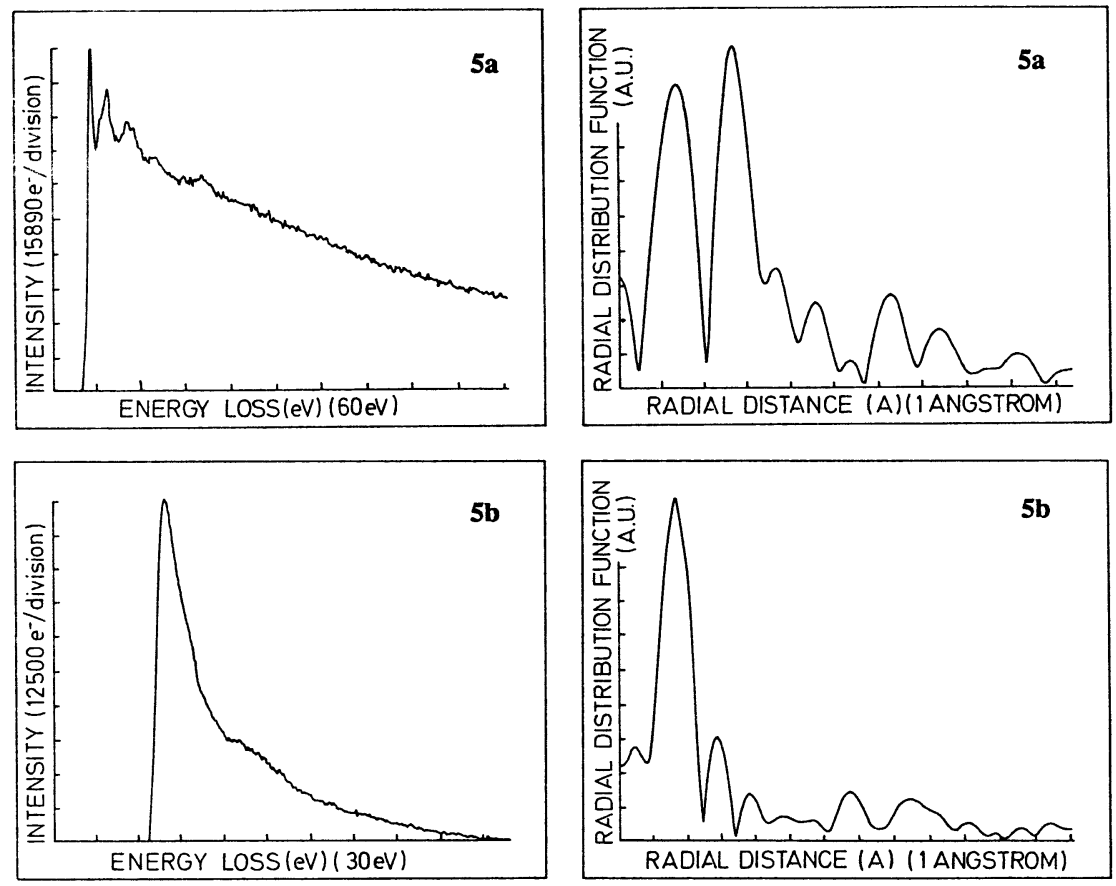

Fig. 5. - Si K edge in crystalline (5a) and amorphous (5b) SiC with the associated RDF.

The resolution limit of the present EXELFS analyses is $0.05 \AA$ as based on the $k$ range and the methods of analysis. An uncertainty in the threshold position $E_{0}$ can also cause shifts in the position of peaks in the RDF. A variation of $5 \mathrm{eV}$ in $E_{0}$ results in corresponding shifts in the position of peaks in the RDF of $0.06 \AA$ for the first (Si-C) peak and $0.07 \AA$ for the second (Si$\mathrm{Si})$ peak. These values are lower than the variations measured from the RDF. For the first peak the RDF shows a $0.16 \AA$ difference in the $\mathrm{Si}-\mathrm{C}$ interatomic distance between crystalline SiC and ion implanted amorphous material. The difference is $0.24 \AA$ in the Si-Si distance and $0.30 \AA$ in the $\mathrm{C}-\mathrm{C}$ distance. These results indicate a relative expansion of the amorphized material. Also the intensity of the second ( $\mathrm{Si}-\mathrm{Si}$ ) peak normalized to the first $\mathrm{Si}-\mathrm{C}$ peak is much lower for the 
amorphous material than for the crystalline $\mathrm{SiC}$. This difference may be due to a change in the coordination number for the $\mathrm{Si}-\mathrm{Si}$ bond. Clearly, even in amorphous ion implanted $\mathrm{SiC}$ the Si$\mathrm{C}$ bond is not completely randomized although its spacing appears to be increased. Also, the nearest neighbor tetrahedral bonds still exists. However, the unit cell structure appears to have been damaged as evidenced by the relative decrease in the Si-Si bond intensity.
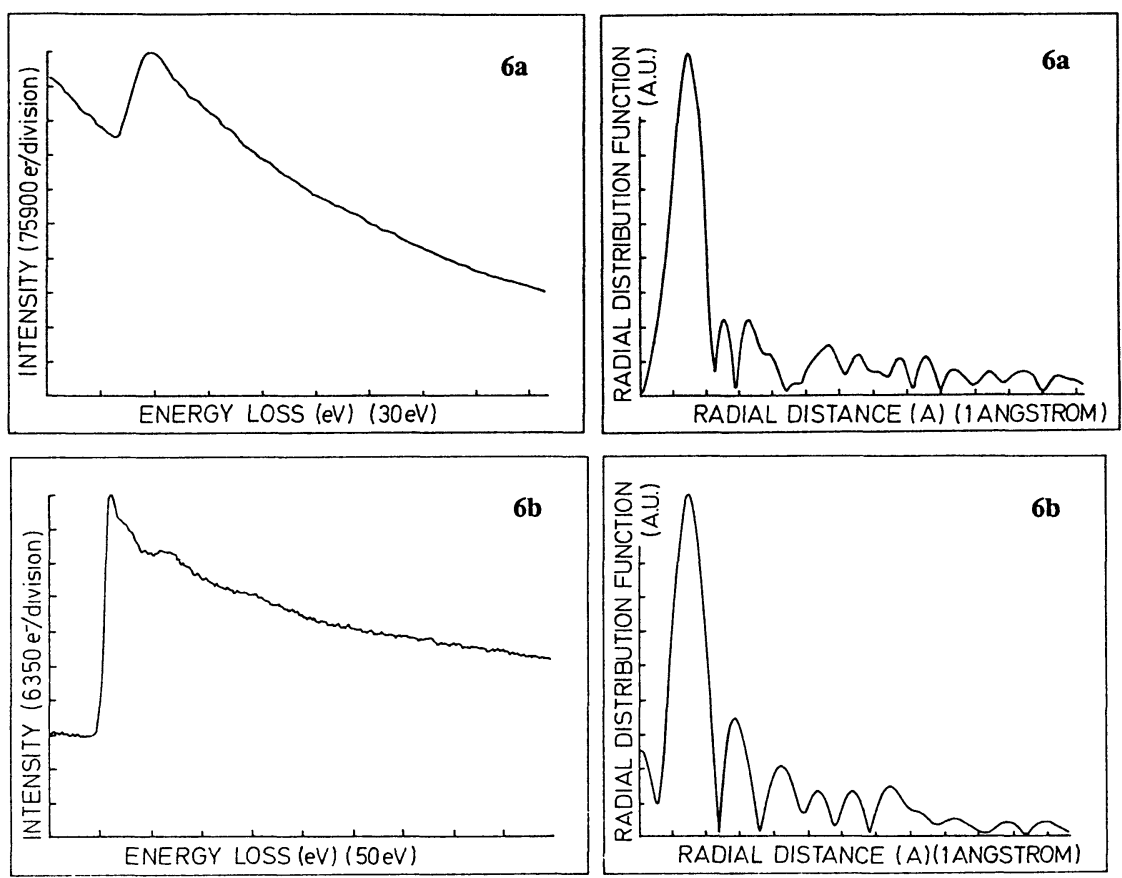

Fig. 6. - CK edge in the crystalline (6a) and amorphous (6b) $\mathrm{SiC}$ with the associated RDF.

\section{Alloys-superalloys}

Many materials are designed and used for their favourable mechanical properties. In order to continuously improve these properties, a deeper and deeper knowledge of the deformation mechanisms of crystals is however necessary, especially those concerning new materials such as light alloys and superalloys.

In contrast with phenomenological studies, which yield empirical equations describing the plastic properties under complex situations, the physical approach is the interpretation of the most simple plastic properties in terms of a set of well characterized microscopic phenomena.

The physical approach appears difficult in the case of complex materials or complex microstructures. It is however easier when the plasticity can be described by the movement of dislocations with constant density $\rho$, and velocity $v$. Under such conditions, the strain rate can be expressed as $\dot{\varepsilon}=\rho b v$, where $b$ is the dislocation's Burgers vector. The problem is thus reduced to the analysis and the interpretation of the velocity of single dislocations. 
This study can be achieved using different complementary techniques. Macroscopic deformation experiments can be performed, yielding quantitative data such as activation energies and areas. These are however not easy to correlate with microscopic processes. Electron microscopy on deformed samples (the so called "post-mortem" observations) can give additional ideas of possible corresponding microscopic processes. They are however highly subjective, since mobile dislocations and their types of movement are difficult to identify in relaxed samples.

Under such conditions, in situ deformation experiments are of fundamental interest, since they combine several advantages of both techniques, i.e. they yield quantitative data on single dislocations, the movement of which is perfectly characterized by direct observation. The technique is briefly described below.

3.1 EXPERIMENTAL - In situ studies are performed either in a $3 \mathrm{MeV}$ or a Jeol 200CX electron microscope. A complete set of straining holders allows us to deform microsamples between $20 \mathrm{~K}$ and $1300 \mathrm{~K}$. They are partly described in [17] and [18].

Microsamples with a thin edged hole at their center are anchored on fixed and mobile jaws. Local values of the stress tensor have been computed in [19]. They well reproduce the macroscopic one at some given areas where observations are performed.

Experiments at $200 \mathrm{kV}$ allow irradiation damage to be eliminated, since they may sometimes be undesirable in HVEM. The remaining problem is however the representativity of deformation experiments in thin foils, since dislocations usually strongly interact with free surfaces. This problem has been discussed in detail recently $[20,21]$. It results that very simple rules can be used, in order to be sure that surface effects play a negligible role:

- the sample thickness has to be much larger than the radius of curvature $R$ of dislocations under the applied stress. This condition is very easily fullfield in high strength materials (small $R)$;

- dislocations not ending at free surfaces (distance $>R$ ) must behave like dislocations ending at free surfaces;

- keeping the same tensile axis and varying the foil plane must lead to consistent results. Several checks are also very useful. For instance: - surface effects are negligible when the velocity of dislocations is proportional to their length (kink nucleation all along the dislocation); - measured microscopic parameters (e.g. the deformation stress) compatible with macroscopic ones ensures similar behaviour;

- a substructure similar to that observed after bulk deformation is also a good indication of similar behaviour.

In short, we consider that even if thin foils present some differences with respect to the bulk material, there is always some degree of similar behaviour, which can be the more easily estimated the more the thickness exceeds $3 R$. This allows us to build a model, which must imperatively be compatible with results of other techniques.

All the results presented below satisfy the above criteria.

3.2 SOME RESUlts ON STRENGTH ANOMALIES In $\mathrm{Be}, \mathrm{Ni}_{3} \mathrm{Al}$ AND SUPERALlOYS. - Several metals and alloys with different crystallographic structures exhibit an anomalous increase of strength at increasing temperature. This stress anomaly is the origin of both the high temperature strength and the low temperature ductility (non-brittleness) of $\mathrm{Ni}_{3} \mathrm{Al}$ and superalloys. However, this important physical process is not quite understood at present [22].

Many different models have been propposed [23-25]. When they are all considered in detail, however they are seen to be very different, mainly because they are based on specific properties of the corresponding crystal structure. This sometimes makes it difficult to transpose them from one structure to another. This point not being very satisfying, it seems reasonable to assume 
that, on the contrary, strength anomalies in various materials result from only one, or very few, mechanisms that may be present in every crystal structure. Starting from this assumption, we have performed a complete study in the simple case of prismatic glide in beryllium and applied the results obtained to the more complex case of cubic glide in the $\mathrm{L}_{12}$ structure and octahedral glide in $\mathrm{Ni}_{3} \mathrm{Al}$.

3.2.1 Prismatic glide in beryllium $[23,24]$. - Dislocation glide in the prismatic planes of beryllium appears to be controlled by a strong interaction between rectilinear screw dislocations and the crystal lattice. However, in contrast to the well known Peierls mechanism, the movement is not continuous but jerky. This has been interpreted in terms of a "locking-unlocking" mechanism [23], acting at all temperatures.

The local stress has been deduced from radii of curvature measurements on non screw segments. It reproduces very well the stress anomaly, which ensures that the physical process of interest is present in the thin foil (Fig. 7a).

Under such conditions, the stress anomaly is interpreted as a consequence of an increase of the dislocation - lattice interaction, through an increase of the non planar spreading of screw dislocations (decrease of stacking fault energy in the basal plane).

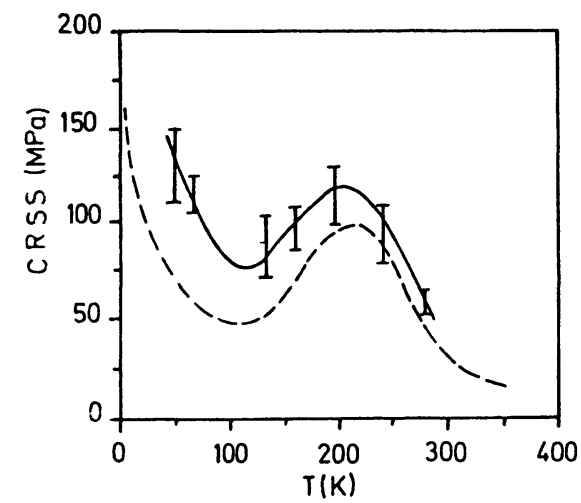

(a)

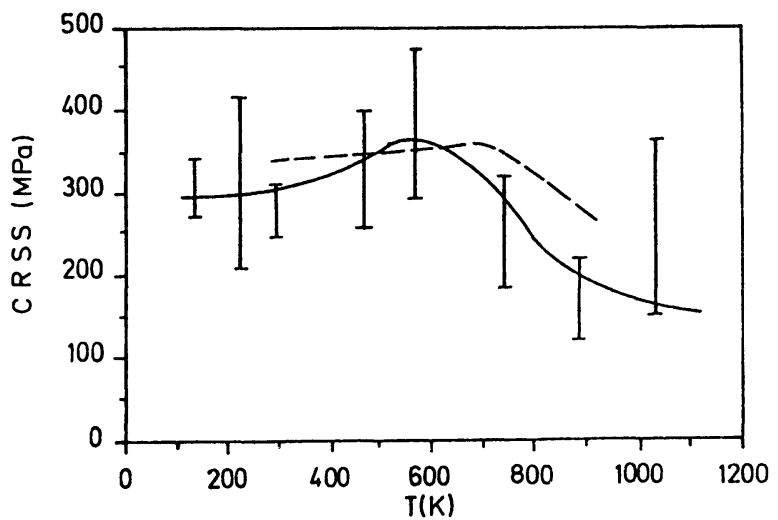

(b)

Fig. 7. - Temperature variation of the critical resolved shear stress (CRSS) of prismatic glide in beryllium (a) and cube glide in the $\gamma^{\prime}$ phase of a superalloy (b). Results from conventional macroscopic experiments (Dotted lines) and from local in situ measurements (Full lines).

3.2.2 Cube glide in the $\gamma^{\prime}$ phase of a superalloy [20, 21, 26]. - A similar behaviour has been observed in this glide system, down to $120 \mathrm{~K}$. In addition, a slight strength anomaly has been evidenced in situ, as in macroscopic experiments (Fig. 7b). This unambiguously proves that the dislocation core is again temperature dependent, which corresponds to a decrease of the so called "complex stacking fault energy" in octahedral planes. This may be due to either a decrease of the corresponding stacking fault energy, or a decrease of the antiphase boundary energy. Dislocation multiplication on polar sources has also been frequently observed (Fig. 8).

3.2.3 Octahedral glide in $\mathrm{Ni}_{3} \mathrm{Al}$ [27]. - In situ experiments performed under weak beam conditions (resolution on mobile dislocations at high temperature: $20 \AA$ ) reveal complex locking- 

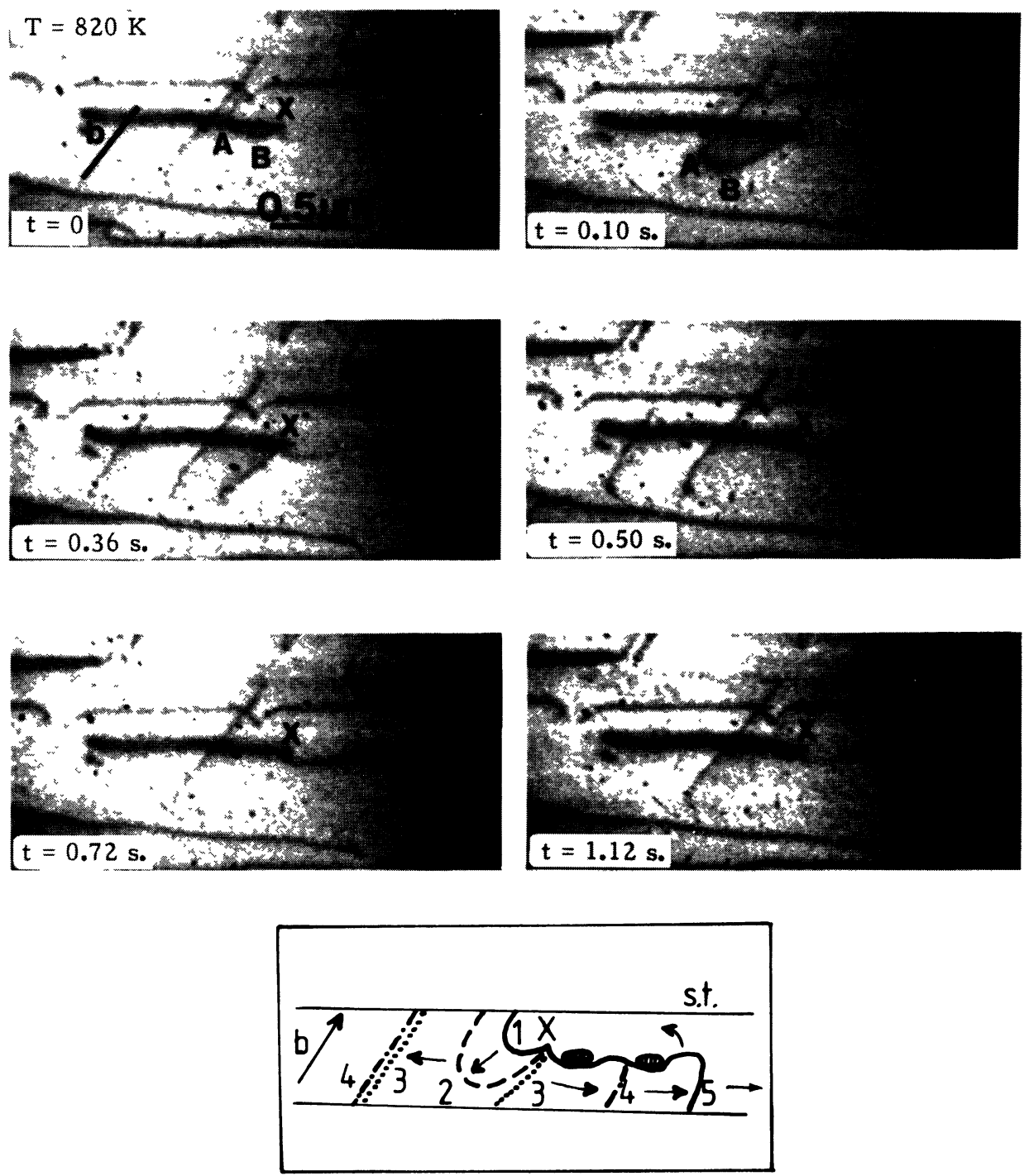

Fig. 8. - Dislocation source in the cube planes of the $\gamma^{\prime}$ phase of a CMSX2 superalloy $(T=820 \mathrm{~K})$. X is the anchoring point and $b$ the direction of the Burgers vector of the dislocation.

unlocking processes, which can be explained only by considering the anisotropy of elastic constants and fault energies. This work is in progress. It however appears that the strength anomaly can be explained in the same way as in the above more simple cases.

3.3 ConClusions. - This new technique yields new information on very important problems which has remained unsolved. It has been used to study the plastic properties of other metals and alloys, and semiconducting compounds. It could a priori be used to study the plastic deformation of many additional non brittle materials. 


\section{Superconductive ceramics.}

4.1 AN "IN SITU" $3 \mathrm{MeV}$ TEM STUDY OF THE REVERSIBLE ORTHORHOMBIC TO TETRAGONAL TRANSITION IN THE SUPERCONDUCTOR ( $\mathrm{Ln}) \mathrm{Ba}_{2} \mathrm{Cu}_{3} \mathrm{O}_{6+x}$. - As it is now well known, the $\mathrm{YBa}_{2} \mathrm{Cu}_{3} \mathrm{O}_{6+x}$ type compounds crystallize in the orthorhombic or in the tetragonal system depending on the value of the oxygen content $(0 \leq x \leq 1)$. More precisely, for $x$ close to 0 , the compound is in the tetragonal phase while the orthorhombic phase is obtained for $x$ close to 1 . This reversible structural transition has already been studied by X-ray or electron diffraction [28, 29]. Depending on the material, the temperature of the phase transition lies around $600-700^{\circ} \mathrm{C}$. When looking at the structures of these compounds it is obvious that the phase transition results from the loss of the oxygen atoms lying in the $\mathrm{CuO}$ planes. It has been shown that the presence of,these atoms is essential for the occurence of a super-normal transition [30,31]. In these compounds, the superconductivity appears under $92 \mathrm{~K}$ for $x \geq 0.62$, that is, in the orthorhombic phase.

The structural phase transition has been studied using the $3 \mathrm{MeV}$ TEM in TOULOUSE, equipped with an environmental cell and a special heating holder. The compound studied, (Ln) $\mathrm{Ba}_{2} \mathrm{Cu}_{3} \mathrm{O}_{6+x}[32,33]$, is isostructural with $\mathrm{YBa}_{2} \mathrm{Cu}_{3} \mathrm{O}_{6+x}$, but presents better morphological characteristics such as bigger grains with clean boundaries and a narrow superconducting transition.

In electron microscopy image mode, the orthorhombic phase is characterized by the presence of domains due to the multiple twinning that occurs during the $\mathrm{T}-\mathrm{O}$ transition.

4.2 EXPERIMENTAL. - The $(\mathrm{Ln}) \mathrm{Ba}_{2} \mathrm{Cu}_{3} \mathrm{O}_{6+x}(0 \leq x \leq 1)$ compound where (Ln) represents a rare earth "cocktail" (Y, Nd, Sm, Eu, Yb) with $20 \%$ of each rare earth, exhibits a $T_{\mathrm{c}}$ of $92 \mathrm{~K}$. The existence of an average ionic radius corresponding to a pseudo rare earth composed by the "cocktail", reported in [32], is in favour of a statistical distribution of the rare earths in the crystal. The experiments have been carried out on the $3 \mathrm{MeV}$ TEM working at an accelerating voltage of $2 \mathrm{MeV}$. With such a high voltage, it has been possible to observe thicker samples than in a conventional microscope. Moreover, this microscope possesses an environmental cell that allows experiments under a controlled gaseous atmosphere [34]. Oxygen pressures less than 120 torrs have been chosen as to limit the diffusion of the electron beam by the gas. Moreover, gas ionisation induced by the beam cannot be avoided. In order to minimize its influence on the measurements the intensity of the electron beam has been kept constant in all the experiments [35]. In this sort of device, the sample is never placed under secondary vacuum so that the risk of contamination is not negligible. The specimen holder used for the experiments is a heating stage where the temperature is measured by a thermocouple chromel-alumel. It is also possible to tilt the sample in order to obtain the best twin contrast.

4.3 RESULTS. - The effect of the oxygen flow pressure on the temperature of the structural phase transition has been investigated. The sample, the starting composition of which is close to the stoichiometry $\mathrm{O}_{7}$, is fixed on the heating stage and inserted in the environmental cell under a selected oxygen pressure. The temperature of the sample is then increased until the twins disappear. Let us call $T_{1}$ the corresponding temperature. The sample is then slowly cooled until the domains appear again: the new temperature being noted $T_{2}$. As during our experiments all the orientation domains appear at the same time, $T_{2}$ is very well defined. The whole process is repeated several times in order to obtain the smaller interval $\left[T_{1}, T_{2}\right]$. The highest accuracy in the determination of the transition temperature is of course obtained when $T_{1}$ is equal to $T_{2}$, but unfortunately our experimental device never gave such an accuracy. 
These experiments have been reproduced at the successive oxygen pressures $45,50,70,80$ and 100 torrs of oxygen. The results are reported in figure 9 where an increase of the transition temperatures with increasing pressures of oxygen around the sample is clearly observed. The general aspect of the graph suggests that above a certain oxygen pressure, the transition temperature remains constant and at its higher value.

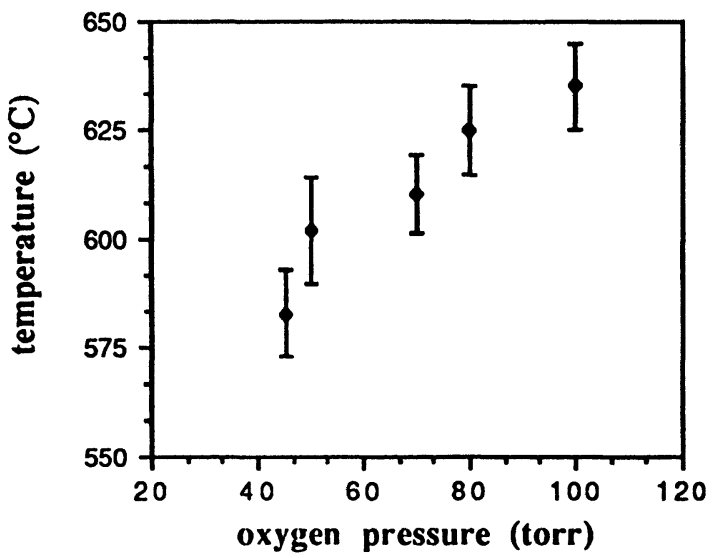

Fig. 9. - Graph of the structural transition temperature versus oxygen pressure.

4.4 Discussion. - Chemisorption at the surface, diffusion in the lattice and fixation on the $O$ (4) sites are the different mechanisms involved in the oxygen absorption and desorption phenomena in $(\mathrm{Ln}) \mathrm{Ba}_{2} \mathrm{Cu}_{3} \mathrm{O}_{6+x}$ compounds. Depending on the thermodynamical conditions, one of these mechanisms can prevail over the others. The experimental device available on the $3 \mathrm{MeV}$ TEM has enabled us to study the kinetics of the phase transition in ( $\mathrm{Ln}$ ) $\mathrm{Ba}_{2} \mathrm{Cu}_{3} \mathrm{O}_{6+x}$ compound. It has then been possible to point out the effect of the pressure on the T-O transition temperature. It is probable that, with increasing pressure around the sample, a maximum of the transition temperature will be attained. Then, analysing the kinetics of the annealing process under oxygen flow, we have found that the compound presents two different behaviours depending on the temperature. Below a temperature which lies around $560^{\circ} \mathrm{C}$ the diffusion phenomenon prevails. Above this temperature, a more and more difficult chemisorption at the surface, and a delocalisation of the oxygen among the different $O(4)$ sites increase the measured times. Of course, at higher temperatures the time required for the transition to occur becomes infinite because only the tetragonal phase is stable. The same behaviour has been observed for different pressures of oxygen around the sample. The reported experiments have pointed out the existence of an optimum temperature of annealing under oxygen which lie around $560^{\circ} \mathrm{C}$.

4.5 INFLUENCE OF THE PARTIAL SUBSTITUTION OF SMALL RARE EARTHS FOR Ca IN THE SUPERCONDUCTIVE Bi-Sr-Ca-Cu-O COMPOUND. - Chemical substitutions have already provided some useful information about the part played by the different elements in the remarkable properties of new high $T_{c}$ superconductors $[36,37]$. The results reported in the present paper concerns the influence of the substitution of small rare earths for $\mathrm{Ca}$ on the structure and electrical properties of the 2:2:1:2 phase in the Bi-Sr system. 
Samples corresponding to the composition $\mathrm{Bi}_{2} \mathrm{Sr}_{2} \mathrm{Ca}_{1-y} \mathrm{Ln}_{y} \mathrm{Cu}_{2} \mathrm{O}_{8+x+y / 2}$ with $0 \leq y \leq 1$ were prepared using the starting materials $\mathrm{Bi}_{2} \mathrm{O}_{3}, \mathrm{CuO}, \mathrm{Ln}_{2} \mathrm{O}_{3}(\mathrm{Ln}=\mathrm{Yb}, \mathrm{Er}), \mathrm{SrCO}_{3}, \mathrm{CaCO}_{3}$ in stoichiometric ratios. Single crystals were grown using a flux method. The samples including $\mathrm{Yb}$ atoms are single phases up to a substitution rate of $y=0.5$ while complete substitution $(y=1)$ can be achieved with Er cations.

Black crystals of good quality corresponding to the formula $\mathrm{Bi}_{2} \mathrm{Sr}_{2} \mathrm{Ca}_{1-y} \mathrm{Yb}_{y} \mathrm{Cu}_{2} \mathrm{O}_{8+x+y / 2}$ where $y=0.1$ to 0.5 in steps of $\Delta y=0.1$ and to the formula $\mathrm{Bi}_{2} \mathrm{Sr}_{2} \mathrm{Ca}_{1-y} \mathrm{Er}_{y} \mathrm{Cu}_{2} \mathrm{O}_{8+x+y / 2}$ where $y=0.1$ to $1(\Delta y=0.1)$ have been selected for X-ray and electron microscopy investigations.

4.6 RESULTS. - Electron and X-ray diffraction investigations are consistent with the space group No. 66, Cccm (proposed for instance in [38]), or Bbmb according to our choice of unit cell axes: long axis along the $c$ direction and the incommensurate component of the modulation wave vector lying in the $b^{*}$ direction. Careful electron diffraction work has been carried out in order to investigate the four-dimensional reciprocal lattice [39]. These compounds present a superconducting transition for $y \leq 0.5$ but exhibit a semiconductor behaviour for higher rare earth content. For instance, the compounds containing $\mathrm{Yb}$ undergo a superconducting transition at $88 \mathrm{~K}$ for $y=0.1,92 \mathrm{~K}$ for $y=0.3$ and at $73 \mathrm{~K}$ for $y=0.4$. Also some structural characteristics of the compounds are affected by the rare earth substitution degree.

Figure 10 shows that the long axis c, continuously decreases with increasing $y$. Whatever the substitution rate, the parameters $a$ and $b$, strongly correlated with the perovskite parameter, do not vary too much within the accuracy of our measurements.

The influence of the $\mathrm{Yb}$ substitution on the period of the modulation is presented in figure 11.

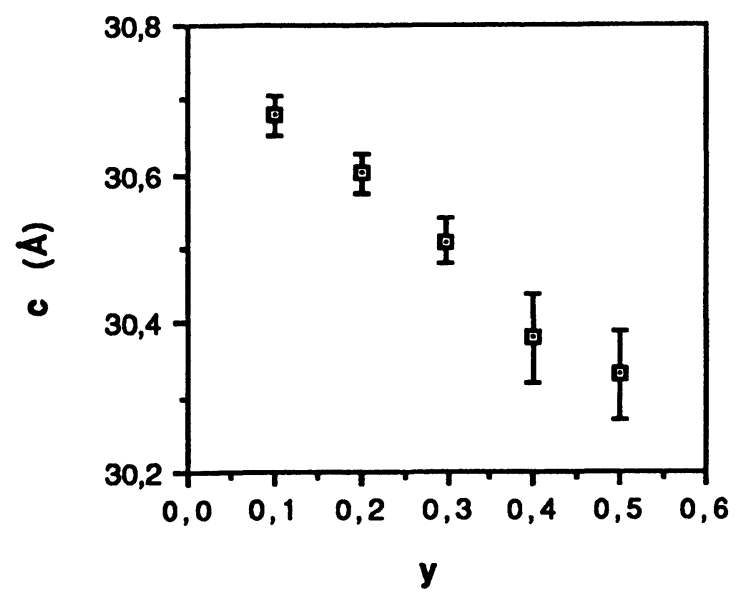

Fig. 10.

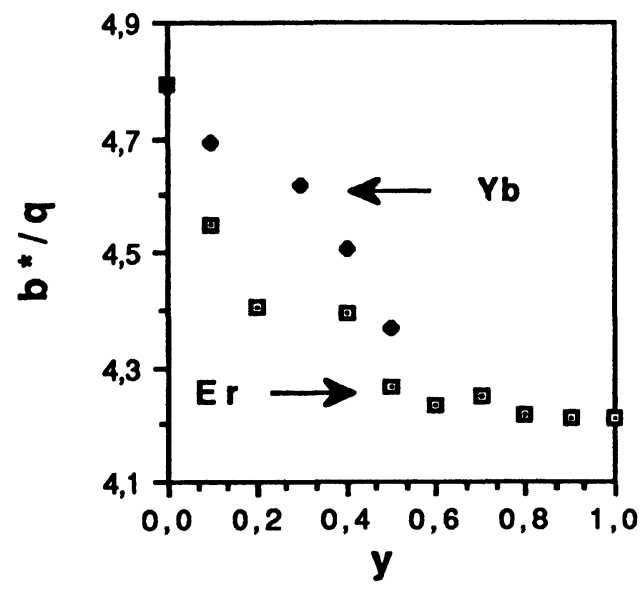

Fig. 11.

Fig. 10. - The unit cell parameter $\mathrm{c}$ as a function of the $\mathrm{Yb}$ content.

Fig. 11. - The modulation period as a function of $\mathrm{Yb}$ content.

High substitution rates $(y>0.5)$ were not possible with Yb. From these plots, it is clear that substituting $\mathrm{Yb}$ (or Er) for $\mathrm{Ca}$ up to $y=0.5$ induces a decrease of the modulation period. Compounds with higher rare earth content, reported in the Er case, present nearly the same $\mathbf{b}^{*} / \mathbf{q}$ 
ratios as the $y=0.5$ compound.

In summary, except for the parameter, the structural and electrical properties present two distinct behaviours for $y \leq 0.5$ and $y>0.5$.

The origin of the incommensurate modulation in the Bi-Sr system has been widely discussed. Many authors consider that additional oxygen inside the $\mathrm{Bi}-\mathrm{O}$ layers are responsible for the modulation. Substitution of $3+$ cations actually modify the oxygen content in order to compensate for the electrical charge. The comparison of the structure of the $y=0$ and $y(\mathrm{Yb})=0.4$ compounds reported in [40] clearly show the presence of addional oxygen atoms (Fig. 12).
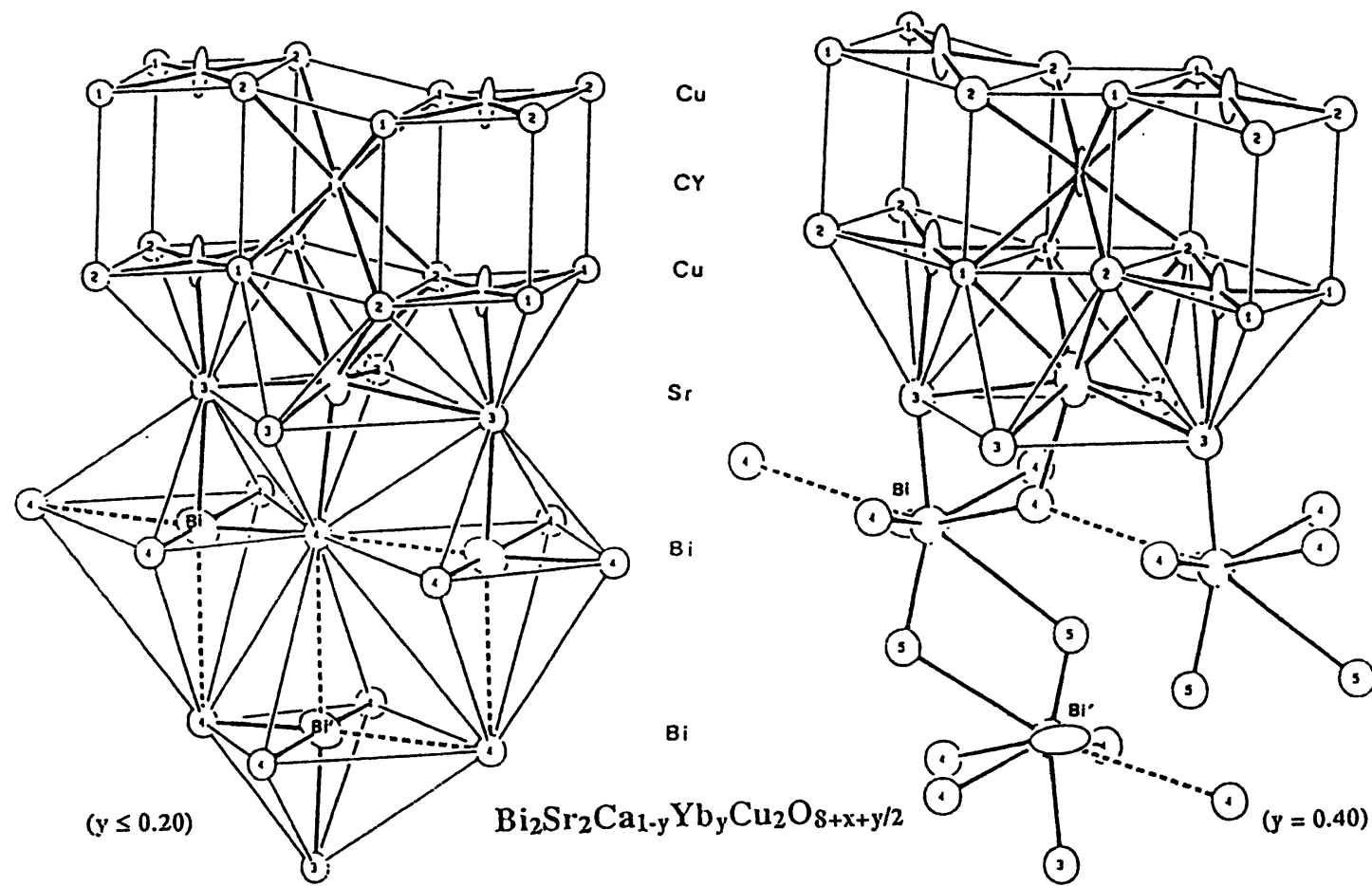

Fig. 12. - Perspective view of the average structure of $\mathrm{Bi}_{2} \mathrm{Sr}_{2} \mathrm{Ca}_{1-y} \mathrm{Yb}_{y} \mathrm{Cu}_{2} \mathrm{O}_{8+x+9 / 2}$ for $y \leq 0.2$ and $y=0.4$.

\section{References}

[1] SHINOZAKI S., and KINSMAN K. R., Acta Metall. 26 (1978) 769.

[2] JeEPs N. W., SMith D. J., PAGe T. F., Acta Cryst. A35 (1979) 916.

[3] ZANCHI G., HsSEIN K., SEVELY J., EMAG 87. Analytical electron microscopy, G. W. Lorimer, Ed. (1988) p. 123.

[4] RAMSDELL L. S., ann. Mineral 32 (1947) 64.

[5] SCHAMM S., MAZEl A., DoRIGNAC D., SEVEly J., Matériaux composites pour application à H.T., R. Naslain et al., eds. (1990) p. 207. 
[6] SPENCE J. H., Experimental High Resolution Electron Microscopy (Clarendon Press, Oxford, 1981).

[7] O'KeEFE M. A., BUSECK P. R., Trans. Am. Crystallogr. Assoc. 15 (1979) 27-46.

[8] EgERTon R. F., Electron Energy Loss Spectroscopy in the Electron Microscope (Plenum Press, N. Y., 1986) p. 262.

[9] EGERTON R. F., Ultramicroscopy 6 (1981) 207.

[10] ZaluZec N. J., 41st Ann. Proc. Electron. Microsc. Sec. Ann., G.N. Bailey Ed. (San Francisco, 1983) p. 388.

[11] Manoubi T., Tence M., Walls M. G., Colliex C., Microsc. Microanal. Microstruct. 1 (1990) 23.

[12] SEVELY J., Electron Microscopy and Analysis (Institute of Physics Conference Series number 78, Ed. G.J. Tatlock) 1985 p. 155.

[13] LeAPMAN R. D., GRUNeS L. A., Fejes P. L., Silcox J., in EXAFS Spectroscopy, B. K. Reo and C. D. Joy (Plenum Press, N. Y., (1981) p. 217.

[14] ANGELINI P., SEVEly J. C., HSSEIN K., ZANCHI G., Analytical electron microscopy, D.C. Joy Ed. (San Francisco Press, 1987) p. 267.

[15] ISSACSON M., JOHNSON D., Ultramicroscopy 1 (1975) 33.

[16] TEO B. K., LEE P. A., J. Am. Chem. Soc. 101 (1979) 2815.

[17] COURET A., Thèse ${ }^{\circ} 490$, Université Paul Sabatier, Toulouse (1989).

[18] Couret A. and CAIllard D., Acta Metall. 36 (1988) 2515.

[19] Coujou A., Lours P., Roy N. A., CaIllaRd D. and Clement N., Acta Metall. 38 (1990) 825.

[20] Clement N., Couret A. and Caillard D., to appear in Philos. Mag. (1991).

[21] Clement N., Molenat G. and Caillard D., to appear in Philos. Mag. (1991).

[22] HiRCH P. B., Proc. Int. Conf. on the Mechanisms of Deformation and the Strength of Advanced Materials, Aussois, to appear in J. Phys. III (1990).

[23] Couret A. and CAILlard D., Philos. Mag. $A$ 59 (1989) 783.

[24] COURET A. and CaIllard D., Philos. Mag. $A 59$ (1989) 801.

[25] POPE D. P. and EZZ S. S., Int. Met. Rev. 29 (1984) 136.

[26] Clement N., Caillard D., Lours P. and Coujou A., Scr. Metall. 23 (1989) 563.

[27] Molenat G. and CaIllard D., to appear in Philos. Mag.(1991).

[28] Hodeau J. L., Chaillout C., CAPponi I. J., MareZio M., Solid State Commun. 64 (1987) 1349.

[29] Schuller Ivan K., Hinks D. G., Beno M. A., CAPONE II, D. W. SODERHOLM L., LOCQUET J. P., BRUYNSERAEDE Y., SEgRE C. U., ZHANG K., Solid State Commun. 63 (1987) 385.

[30] Monod P., Ribault M., D’yvoire F., Jegoudez J., Collin G., Revcolevschi A., J. Phys. France 48 (1987) 1369.

[31] Ourmazd A., Rentschler J. A., Spence J. C. H., O’keeffe M., Graham R. J., Johnson Jr., D. W., RHODES W. W., Nature 327 (1987).

[32] Millet P., Enjalbert R., Galy J., Faulmann C., Cassoux P., Rakoto H., Askenazy S., C. R. Acad. Sci. Paris Ser. II 306 (1988) 407.

[33] Galy J. Enjalbert R., Millet P., Casanove M. J., Roucau C., International Discussion Meeting on High Tc Superconductors, Austria, Feb. 7-11, 1988.

[34] LOCATElli D., MARTinez J. P., BalladORE J. L., Jouffrey B., 6th Int. Conf. on HVEM, Anvers 4 (1980) 74.

[35] Zou J., Cockayne D. J., Auchterlonie G., McKenzie D. R., Dou S. X., Bourdillon A. J., SOREell C. C., EASTERling K. E., JohSON A. W. S., Philos. Mag. Lett. 57 (1988) 157.

[36] Tarascon J. M., Barboux P., Hull G. W., Ramesh R., Greene L. H., Giroud M., Hedge M. S., McKinnon W. R., Phys. Rev. B 39 (1988) 4316.

[37] Zandbergen H. W., Groen W. A., Mulhoff F. C., Van Tendeloo G., Amelinckx S., Physica $C$ 156 (1988) 325.

[38] Subramanian M. A., Torardi C. C., Calabrese J. C., Gopalakrishnan J., Morissey K. G., ASkew T. R., FLIPPEN R. B., CHOWDHRY U., SLEIGHT A. W., Science 239 (1988) 1015.

[39] Casanove M. J., Baules P., Snoeck E., Roucau C., Physica C 159 (1989), 461.

[40] Millet P., SeEger O., EnJalbert R., Galy J., J. Solid. State Chem. 90 (1991) 344. 